\title{
A case of an unusual lineage switch in late relapse ALL-is it actually a secondary leukemia?
}

\author{
Antonija Babić ${ }^{1}$ (D) Lejla Kurić $^{1}$ - Klara Dubravčić ${ }^{1}$ - Zinaida Perić ${ }^{1}$ - Josip Batinić ${ }^{1}$ - Višnja Armanda ${ }^{2}$. \\ Dubravka Kuljiš $^{2}$ - Drago Batinić ${ }^{1}$
}

Received: 9 April 2019 / Accepted: 11 September 2019 / Published online: 20 November 2019

(C) Springer-Verlag GmbH Germany, part of Springer Nature 2019

\begin{abstract}
Acute lymphoblastic leukemia (ALL) is a malignant disease of lymphoid precursors. According to immunophenotype, it is further subdivided into precursor B cell ALL and precursor T cell ALL, with precursor B cell ALL being much more common both in children and adults. Lineage switch from one lymphoid lineage to another during the course of the disease is extremely rarely reported. Here, we describe a case of a child who initially presented as a precursor B-ALL but 15 years later and after two successfully treated relapses of the original ALL presented with early T cell precursor leukemia. Although it was considered as a relapse, it could be interpreted as a case of secondary leukemia, which can be explained as a consequence of treatment as well as a constitutional feature of an individual. Also, it draws attention to the possibility that hematopoietic cells, and in that context also leukemic cells, are much more plastic and capable of reprogramming than previously thought.
\end{abstract}

Keywords Acute lymphoblastic leukemia $\cdot$ Secondary leukemia $\cdot$ Lineage switch

\section{Introduction}

Acute leukemias are the most common type of cancer in children and the majority of them are acute lymphoblastic leukemia (ALL). According to their immunophenotype, they are further divided into B-lymphoblastic ( $80 \%)$ and Tlymphoblastic leukemia (15\%) [1]. Conversion of a leukemia clone is a rare event in acute leukemia that is described in about $6-9 \%$ of all relapses [2]. "Lineage switch" is a term used to describe that phenomena in which upon relapse, acute leukemia meets the criteria for the opposite lineage compared with that of the initial diagnosis [3]. Here, we describe the unusual course of the disease of a young male who was diagnosed with a B-ALL in his early childhood, who had a late second relapse of his original leukemia clone in his cervical

Antonija Babić

ababic@kbc-zagreb.hr

1 Department of Laboratory Immunology, Clinical Department of Laboratory Diagnostics, University Hospital Centre Zagreb and University of Zagreb School of Medicine, Kišpatićeva 12, 10000 Zagreb, Croatia

2 Department of Pediatrics, Hematology-Oncology Unit, University Hospital Centre Split, 21000 Split, Croatia lymph nodes 15 years after the initial diagnosis with a molecular infiltration of the bone marrow, followed by a third bone marrow relapse 2 years after, with a conversion of a leukemia clone to that of early $\mathrm{T}$ precursor.

\section{Case report}

The patient was initially diagnosed as having acute leukemia in 1999 at the age of 2 years (Fig. 1). His bone marrow sample was collected in $\mathrm{K}_{3}$-EDTA 3.0-mL plastic tubes (Becton Dickinson Biosciences, San Jose, USA) and sent to our laboratory for flow cytometry (FCM) immunophenotyping that revealed a typical B-precursor "common"-ALL with CD13 co-expression $\left(\mathrm{CD} 19^{+}, \mathrm{CD} 10^{+}, \mathrm{CD} 13^{+}, \mathrm{CD} 34^{+}, \mathrm{HLA}^{-} \mathrm{DR}{ }^{+}, \mathrm{CD} 7^{-}\right.$, cytoplasmatic (cyt) $\mathrm{IGM}^{-}$, membrane (mem) $\mathrm{IGM}^{-}, \mathrm{CD}^{+} 3^{+}$, $\mathrm{CD}^{-} 4^{-}, \mathrm{MPO}^{-}$). The boy was treated according to ALL-BFM 95 protocol. On day 15 of the treatment, a bone marrow contained $6 \%$ residual leukemia cells by flow cytometry (FCM) and on day 33, he was in a complete remission, without measurable minimal residual disease (MRD) by FCM. After that, he was without any sign of the disease for more than 3.5 years. However, in January 2003, the patient developed relapse with bone marrow FCM analysis showing $49 \%$ of blasts of the original immunophenotype $\left(\mathrm{CD} 19^{+}, \mathrm{CD} 10^{+}, \mathrm{CD} 34^{+}\right.$, 
$\left.\mathrm{CD} 13^{+}\right)$. He then started the treatment according to AIEOP-LLA REC 98 protocol and after 2 months of treatment, the bone marrow FCM MRD was $0.03 \%$, but after 3 months of therapy, there were no immunophenotipically detectable residual blasts in the bone marrow. Maintenance therapy was completed in 2006 and the patient had no clinical signs of the disease for another 8 years. At the age of 17 , he was admitted to a hospital with swelling in the left side of his neck. Detailed examination established a pack of lymph nodes along the sternocleidomastoid muscle and a fine needle aspiration was performed. Cytomorphological examination was suggestive of lymphoblastic lymphoma and biopsy confirmed that the histology (large blast looking lymphatic cells with vesicular nucleus and visible nucleolus) and immunohistochemistry $\left(\mathrm{CD} 79 \mathrm{a}^{\text {low }}, \mathrm{CD} 10^{+}\right.$, $\mathrm{CD}^{4} 4^{+}, \mathrm{CD}_{3} 3^{+}, \mathrm{CD} 9^{+}, \mathrm{BCL}^{+}, \mathrm{CD} 20^{-}, \mathrm{CD}^{-}, \mathrm{CD}^{-}{ }^{-}$, cyclin $\mathrm{D}^{-}, \mathrm{BCL}^{-}, \mathrm{TdT}, \mathrm{CD}^{-}, \mathrm{CD}^{-}, \mathrm{CD} 23^{-}, \mathrm{CD} 4^{-}$) matched a relapse of the previously diagnosed B-lymphoblastic leukemia/ lymphoma. Parallel examination of the bone marrow aspirate by cytomorphology, immunophenotyping, and cytogenetics (normal 46XY karyotype) did not reveal any signs of leukemia cells, except for the polymerase chain reaction (PCR) analysis showing $\mathrm{t}(12 ; 21)$ type TEL/AML1 positivity. He was given another cycle of therapy according to ALL-REZ BFM 2002 protocol with the addition of anti-CD20 (rituximab). Till the beginning of 2015, the patient received 4 doses of rituximab and was admitted to a hospital for the next cycle of therapy according to the protocol, but developed acute pancreatitis. The chemotherapy was discontinued and a surgical procedure with necrectomy of the pancreas was done after which the patient was released from the hospital. PET scan done in August 2015 showed no signs of malignant disease. In September 2016, he was admitted to the hospital as febrile neutropenia and bone marrow cytomorphology suggested relapse of the disease with $5 \%$ blasts in the cytology report. PET scan showed signs of metabolically active disease in left cervical lymph nodes. Lymph node biopsy showed medium-sized atypical lymphocytes that on immunohistochemistry were positive for cytCD3, CD5, CD7, CD34, and CD99 and negative for CD2, CD4, CD8, CD20, PAX5, and MPO which led to diagnosis of lymphoblastic T-lymphoma. Microscopic examination of the second bone marrow aspirate revealed numerous (92\%) agranular and mostly small blasts with some larger blasts that had convoluted nuclei and a part of the blasts that showed typical "hand-mirror "image. Cytochemistry showed partial dot-like positive staining for $\alpha$-naphthyl acetate esterase ( $\alpha$ NAE) which was blocked by sodium fluoride, while myeloperoxidase (MPO), Sudan black, chloroacetate esterase (CAE), and periodic acid-Schiff (PAS) were all negative. The FCM analysis showed $\mathrm{T}$ lineage blasts $\left(\mathrm{cytCD} 3^{+}, \mathrm{TdT}^{\mathrm{dim}}\right.$, memCD $3^{\text {neg }}, \mathrm{CD}^{\mathrm{dim}}, \mathrm{CD}^{+}, \mathrm{CD} 10^{+}, \mathrm{CD} 34^{+}$) with coexpression of myeloid markers CD13, CD33, and CD117. This led us to conclude the patient developed an early Tlymphoblastic leukemia (ETP). Cytogenetic fluorescence in situ hybridization (FISH) analysis as well as PCR analysis showed no positivity for the previously found $t(12 ; 21)$ and TEL/AML1 fusion gene. Karyotyping was not done due to low mitotic index. Reinduction was done with HAM protocol and complete cytomorphological remission with no MRD by FCM was achieved. In January 2017, the patient received allogeneic hematopoietic stem cell transplant (HSCT) from an unrelated donor. After 22 months, he is still in complete remission (CR).

\section{Materials and methods}

Bone marrow samples were analyzed by four-color flow cytometry (Figs. 1 and 2). The antibodies and clones that were examined are shown in Table 1. CD1a, CD10, CD79a, MPO, TdT, and lysozyme antibodies were purchased from Dako (Santa Clara, USA) and CD5, CD8, CD20, CD4, CD7, CD10, CD13, CD33, CD117, CD7, CD45, HLA-DR, CD34, CD3, CD15, and CD19 antibodies from Becton Dickinson (BD) Biosciences (San Jose, USA).

One hundred microliters $\left(1 \times 10^{6}\right.$ cells $)$ of bone marrow per tube were incubated for $15 \mathrm{~min}$ with flourochrome conjugated antibodies according to manufacturer's recommendations and afterwards, erythrocytes were lysed with BD FACS Lysing solution (BD Biosciences, San Jose, USA). Finally, samples were washed once in phosphate-buffered saline (PBS) and suspended in $300 \mathrm{~mL}$ of PBS. The FACS Calibur (BD Biosciences, San Jose, USA) was used for cell analysis. Data were analyzed by FACS CellQuest Pro version 5.2.1 (BD Biosciences, San Jose, USA).

\section{Discussion}

Most relapses of childhood acute lymphoblastic leukemia occur during the time of treatment or within 2 years after treatment. They are considered early relapses in contrast to late relapses defined according to different study groups as those occurring at least 30 months or 3 years after initial diagnosis [4]. The most accepted theory for relapse is the persistence of a small undetectable malignant clone, resistant to applied chemotherapy regime [5]. However, late relapses can represent a completely new clone characteristic of secondary leukemia in a patient with a history of previous malignancy [6] who received cytotoxic treatment [7].

Since most often secondary leukemias are of myeloid lineage, secondary AML is well known and described, with secondary lymphoblastic leukemia mentioned in only $5-10 \%$ of cases [8]. Rosenberg et al. made a distinction between patients who had received treatment for their first malignancy (therapy-associated ALL, t-ALL) and those who had not (antecedent malignancy ALL, am-ALL) [6]. They, as well as some other authors, suggest that secondary acute lymphoblastic leukemia is constitutional and probably not related to prior 


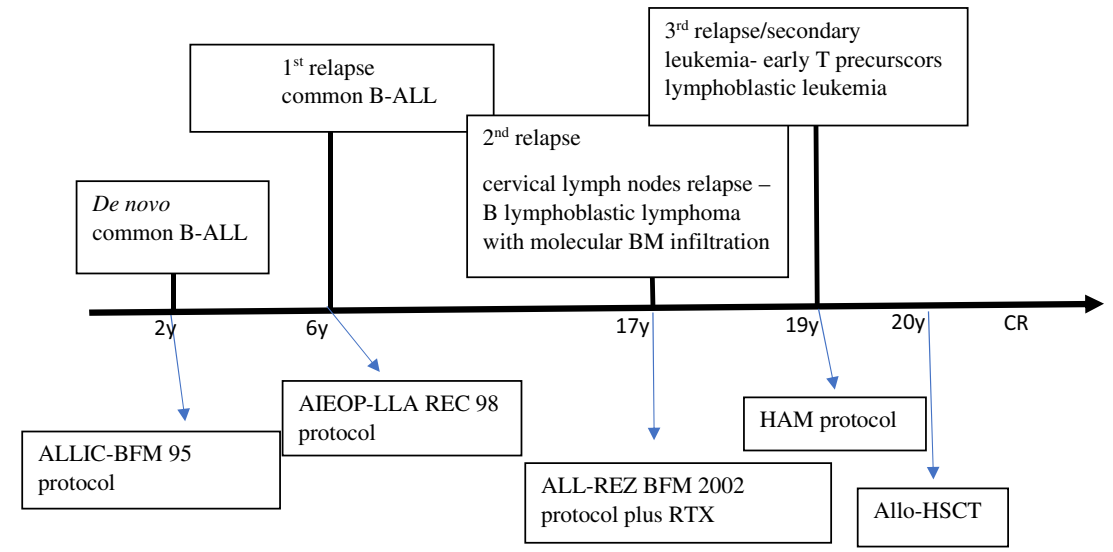

Fig. 1 Flowchart of events

therapy [9]. In other words, s-ALL patients may have an inherent predisposition to malignancies and a history of previous therapy may be of lesser importance in the pathogenesis of s-ALL. Secondary lymphoblastic leukemia is more and more recognized as a distinct entity from de novo ALL and carries adverse prognostic implications that vary by age. It is of particular relevance in the pediatric/adolescent/young adult (AYA) population, in whom ALL would be otherwise quite treatable with a high rate of cure [6].

However, these studies on secondary ALL exclude patients with a prior primary acute leukemia $[6,9]$ due to difficulties in distinguishing it from a relapse. Therefore, a secondary ALL after a prior diagnosis of ALL is extremely rarely reported. Zuna et al. [10] tried to establish diagnostic criteria for "secondary ALL after ALL" by comparing clonal markers-Ig/TCR rearrangement, fusion proteins, and immunophenotype at the initial diagnosis and recurrence of leukemia. According to that criteria, for the diagnosis of secondary lymphoblastic leukemia, there should be no clonal connection (Ig/TCR rearrangement, cytogenetic marker, or fusion gene) between the initial diagnosis and recurrence of the leukemia. There should also be at least one of the B-criteria, which includes a significant immunophentoype shift (most often a lineage switch), cytogenetic shift, and molecular shift (loss or gain of a fusion gene).
Fig. 2 Flow cytometry analysis of leukemic early $\mathrm{T}$ precursors in 3rd relapse with characteristic immunophenotype (cytCD3+ Tdtdim CD5dim/neg CD7+ CD19neg CD34+ CD117+ CD13dim+; antigen expression rating according to AIEOP-BFM guidelines)
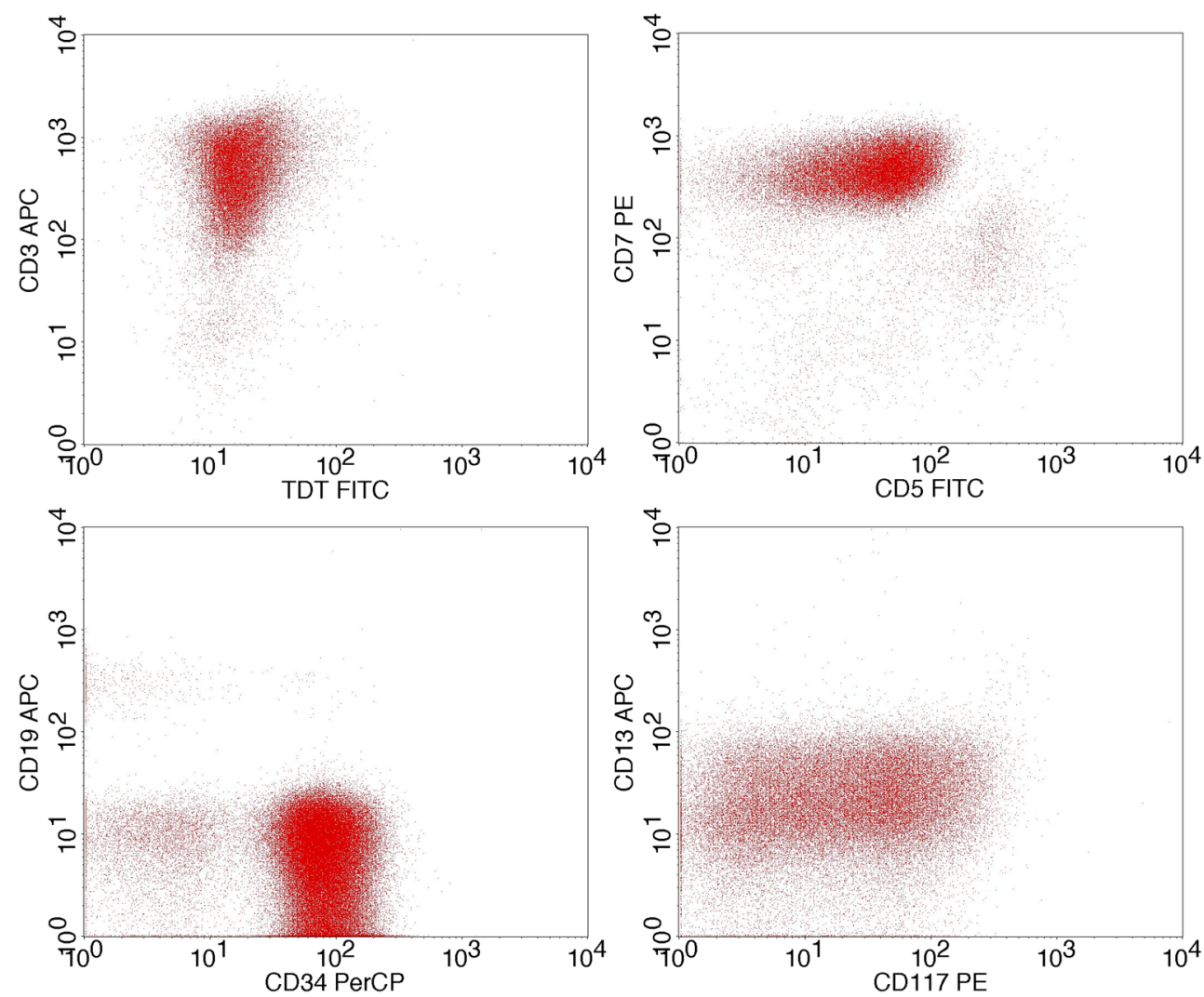
Table 1 Antibodies and clones that were examined

\begin{tabular}{ll} 
Fluorochrome & Antibody (clone) \\
\hline FITC & $\begin{array}{c}\text { CD1a (NA1/34), CD5 (L17F12), CD8 (SK1), CD20 } \\
\text { (L27), TdT(HT6), Lysozyme (polyclonal) }\end{array}$ \\
PE & CD4 (SK3), CD7 (M-T701), CD10 (SS2/36), CD13 \\
& (L138), CD33 (P67.6), CD117 (104D2), CD79a \\
& (HM57), MPO (MPO-7) \\
PerCP & CD45 (2D1), HLA-DR (L243) \\
PerCP-Cy5.5 & CD34 (8G12) \\
APC & CD3 (SK7), CD15 (HI98), CD19 (SJ25C1) \\
\hline
\end{tabular}

In recent years, there are more new concepts trying to explain the mechanism of lineage conversion or so-called "lineage switch" in relapse.

In a classical model of a hierarchical hematopoietic system, cells are conventionally classified into two major lineages, lymphoid and myeloid. However, a number of recent studies indicate that lymphoid-myeloid divergence is less abrupt than previously believed [1]. The presence of bipotential hematopoietic progenitors, like B-macrophage progenitor, has raised the possibility that if a leukemogenic event occurs in that type of early potential progenitor, the cell could differentiate into very different cell populations. This hypothesis is also used to explain mixed phenotype acute leukemia (MPAL). On the other hand, hematopoietic cell development might be more flexible than previously thought, with potential to be reversible and multidirectional [11]. A network of transcriptional factors controls the development and lineage commitment of hematopoietic cells, but genetic and epigenetic changes in a cell can influence transcription factors responsible and induce reprogramming of a cell lineage and return to a more primitive multipotent stage [1]. For example, enforced expression of myeloid transcription factor $\mathrm{C} / \mathrm{EBP} \alpha$ into $\mathrm{B}$ and $\mathrm{T}$ cells has shown that they can be converted into functional macrophages $[12,13]$. Expression of GATA-1 was able to induce common $\mathrm{B}$ and $\mathrm{T}$ progenitor cells to reprogram into megakaryocytic/ erythroid cells [14]. Also, Somasundaram et al. [11] showed that pro-B leukemia cells from mice carrying heterozygous mutations in either or both the Pax5 and Ebfl genes, which are crucial for B cell commitment and also commonly mutated in human leukemia, can be converted into $\mathrm{T}$ lineage leukemia cells in vitro.

A third concept is the so-called clonal selection, which describes the existence of a small undetectable sub-clone of different lineage at the initial diagnosis. That minor resistant sub-clone can cause the relapse after the successful treatment of the dominant leukemia clone.

In the case of our patient, there is a lack in medical records at the initial diagnosis 19 years ago, covering molecular and cytogenetic analysis, so we could only follow the immunophenotype throughout the complete course of the disease. According to immunophenotypic analysis, the first relapse after 3.5 years matched the original common B-ALL clone. Second, late relapse in the cervical lymph nodes also had histological and immunohistochemical characteristics of B lymphoblasts, with molecular detection of TEL/AML1 fusion gene in the bone marrow confirming the diagnosis of molecular relapse of B-ALL. However, the third relapse showed a significant shift in the immunophenotype with a lineage switch to early T-ALL and loss of earlier detected TEL/AML1 gene; therefore, we could assume that according to the abovementioned criteria, it was actually a case of secondary ALL.

In conclusion, whether it is a rise of a completely new leukemic clone or a consequence of hematopoietic cell plasticity, the occurrence of a different lineage of leukemia cells during the course of leukemia is a rare but important phenomenon with implications for treatment selection. This also has the potential to expand our knowledge on hematopoietic cell development and pathogenesis of acute leukemia.

\section{Compliance with ethical standards}

Conflict of interest The authors declare that they have no conflict of interest.

\section{References}

1. Dorantes-Acosta E, Pelayo R (2012) Lineage switching in acute leukemias: a consequence of stem cell plasticity? Bone Marrow Res 2012:1-18

2. Ruiz-Delgado GJ, Nuñez-Cortez AK, Olivares-Gazca JC, Fortiz YC, Ruiz-Argüelles A, Ruiz-Argüelles GJ (2017) Lineage switch from acute lymphoblastic leukemia to myeloid leukemia. Med Univ 19(74):27-31

3. Park M, Koh KN, Kim BE, Im HJ, Jang S, Park CJ, Chi HS, Seo JJ (2011) Lineage switch at relapse of childhood acute leukemia: a report of four cases. J Korean Med Sci 26(6):829-831

4. Locatelli F, Schrappe M, Bernardo ME, Rutella S (2012) How I treat relapsed childhood acute lymphoblastic leukemia? Blood J 120(14):2807-2817

5. Choi S, Henderson MJ, Kwan E, Beesley AH, Sutton R, Anita Y et al (2013) Relapse in children with acute lymphoblastic leukemia involving selection of a preexisting drug-resistant subclone. Blood. 110(2):632-639

6. Rosenberg AS, Brunson A, Paulus JK, Tuscano J, Wun T, Keegan THM et al (2017) Secondary acute lymphoblastic leukemia is a distinct clinical entity with prognostic significance. Blood Cancer J 7(9):1-6

7. Hijiya N, Ness KK, Ribeiro RC, Hudson MM (2009) Acute leukemia as a secondary malignancy in children and adolescents: current findings and issues. Cancer. 115(1):23-35

8. Szczepański T, Willemse MJ, Kamps WA, Van Wering ER, Langerak AW, Van Dongen JJM (2001) Molecular discrimination between relapsed and secondary acute lymphoblastic leukemia: proposal for an easy strategy. Med Pediatr Oncol 36(3):352-358

9. Ganzel C, Devlin S, Douer D, Rowe JM, Stein EM, Tallman MS (2015) Secondary acute lymphoblastic leukaemia is constitutional and probably not related to prior therapy. Br J Haematol 170(1):50 55 
10. Zuna J, Cavé H, Eckert C, Szczepanski T, Meyer C, Mejstrikova E, Fronkova E, Muzikova K, Clappier E, Mendelova D, Boutard P, Schrauder A, Sterba J, Marschalek R, van Dongen JJM, Hrusak O, Stary J, Trka J (2007) Childhood secondary ALL after ALL treatment. Leukemia. 21(7):1431-1435

11. Somasundaram R, Åhsberg J, Okuyama K, Ungerbäck J, Lilljebjörn H, Fioretos T, Strid T, Sigvardsson M (2016) Clonal conversion of B lymphoid leukemia reveals cross-lineage transfer of malignant states. Genes Dev 30(22):2486-2499

12. Laiosa CV, Stadtfeld M, Xie H, de Andres-Aguayo L, Graf T (2006) Reprogramming of committed $\mathrm{T}$ cell progenitors to macrophages and dendritic cells by $\mathrm{C} / \mathrm{EBP} \alpha$ and PU.1 transcription factors. Immunity. 25(5):731-744

13. Xie H, Ye M, Feng R, Graf T (2004) Stepwise reprogramming of B cells into macrophages. Cell. 117(5):663-676

14. Iwasaki H, Mizuno SI, Arinobu Y, Ozawa H, Mori Y, Shigematsu H, Takatsu K, Tenen DG, Akashi K (2006) The order of expression of transcription factors directs hierarchical specification of hematopoietic lineages. Genes Dev 20(21):3010-3021

Publisher's note Springer Nature remains neutral with regard to jurisdictional claims in published maps and institutional affiliations. 